\title{
Hurwitz Zeta Function of Two Variables and Associated Properties
}

\author{
M. A. Pathan ${ }^{1}$, Maged G. Bin-Saad ${ }^{2}$ and J. A. Younis ${ }^{3}$ \\ ${ }^{1}$ Centre for Mathematical Sciences, Peechi P.O., Kerala-680653, India \\ e-mail: mapathan@gmail.com \\ ${ }^{2}$ Department of Mathematics, Aden University, Kohrmakssar P.O. Box 6014, Yemen \\ e-mail: mgbinsaad@yahoo.com \\ ${ }^{3}$ Department of Mathematics, Aden University, Kohrmakssar P.O. Box 6014, Yemen \\ e-mail: ali.jihadalsaqqaf@gmail.com
}

\begin{abstract}
The main objective of this work is to introduce a new generalization of Hurwitz-Lerch zeta function of two variables. Also, we investigate several interesting properties such as integral representations, operational connections and summation formulas.
\end{abstract}

\section{Introduction}

The Exton hypergeometric function $H_{E: G ; M ; N}^{A: B ; C ; D}$ of two variables [9, p.339 (13)] is defined by

$$
\begin{aligned}
& \left.H_{E: G ; M ; N}^{A: B ; D}\left[\begin{array}{c}
\left(a_{A}\right):\left(b_{B}\right) ;\left(c_{C}\right) ;\left(d_{D}\right) ; \\
\left(e_{E}\right):\left(g_{G}\right) ;\left(m_{M}\right) ;\left(n_{N}\right) ;
\end{array}\right], y\right] \\
= & \sum_{i, j=0}^{\infty} \frac{\left[\left(a_{A}\right)\right]_{2 i+j}\left[\left(b_{B}\right)\right]_{i+j}\left[\left(c_{C}\right)\right]_{i}\left[\left(d_{D}\right)\right]_{j}}{\left[\left(e_{E}\right)\right]_{2 i+j}\left[\left(g_{G}\right)\right]_{i+j}\left[\left(m_{M}\right)\right]_{i}\left[\left(n_{N}\right)\right]_{j}} \frac{x^{i}}{i !} \frac{y^{j}}{j !}, \quad(0<|x|<1,0<|y|<1),
\end{aligned}
$$

Received: November 13, 2019; Accepted: March 3, 2020

2010 Mathematics Subject Classification: 33B99, 33C15, 33C45, 33C60.

Keywords and phrases: Exton's double hypergeometric function, Hurwitz-Lerch zeta functions, MellinBarnes integrals, operational connections, summation formulas.

Copyright (C) 2020 Maged G. Bin-Saad et al. This is an open access article distributed under the Creative Commons Attribution License, which permits unrestricted use, distribution, and reproduction in any medium, provided the original work is properly cited. 
where for the sake of convenience (in the contracted notation), $\left(a_{A}\right)$ denotes the array of $A$ parameters given by $a_{1}, a_{2}, \ldots, a_{A}$ in the contracted notation of Slater [17, p.54]. The symbol $\Delta(M ; a)$ represents an array of $M$ parameters $\frac{a}{M}, \frac{a+1}{M}, \ldots, \frac{a+M-1}{M}[21$, p.47, pp.213].

The special case $A=E=0$ in (1.1) reduces to the Kampé de Feriet double hypergeometric function [22, p.423 (26), see also[23, p.23 (1.2,1.3)]

$$
\begin{gathered}
F_{G ; M ; N}^{B ; C ; D}(x, y)=H_{0: G ; M ; N}^{0: B ; C ; D}(x, y)=\sum_{i, j=0}^{\infty} \frac{\left[\left(b_{B}\right)\right]_{i+j}\left[\left(c_{C}\right)\right]_{i}\left[\left(d_{D}\right)\right]_{j}}{\left[\left(g_{G}\right)\right]_{i+j}\left[\left(m_{M}\right)\right]_{i}\left[\left(n_{N}\right)\right]_{j}} \frac{x^{i}}{i !} \frac{y^{j}}{j !}, \\
(0<|x|<1,0<|y|<1) .
\end{gathered}
$$

Similarly, when $B=G=0$, then (1.1) reduce to the double hypergeometric function due to Exton [10, p.137 (1.2)]

$$
\begin{gathered}
X_{E: M ; N}^{A: C ; D}(x, y)=\sum_{i, j=0}^{\infty} \frac{\left[\left(a_{A}\right)\right]_{2 i+j}\left[\left(c_{C}\right)\right]_{i}\left[\left(d_{D}\right)\right]_{j}}{\left[\left(e_{E}\right)\right]_{2 i+j}\left[\left(m_{M}\right)\right]_{i}\left[\left(n_{N}\right)\right]_{j}} \frac{x^{i}}{i !} \frac{y^{j}}{j !}, \\
(0<|x|<1,0<|y|<1)
\end{gathered}
$$

which, for $A=D=E=N=0$ and $y=0$ we shall obtain the generalized hypergeometric function CFM defined by [20, p.19 (23)]

$$
{ }_{C} F_{M}\left[\begin{array}{c}
\left(c_{C}\right) ; \\
\left(m_{M}\right) ;
\end{array}\right]=\sum_{i=0}^{\infty} \frac{\left[\left(c_{C}\right)\right]_{i}}{\left[\left(m_{M}\right)\right]_{i}} \frac{x^{i}}{i !}
$$

where $C$ and $M$ are positive integers or zero; $m_{M} \neq 0,-1,-2, \ldots$. The Hurwitz-Lerch zeta function $\Phi(z, s, a)$ is defined by (see $[8,19])$

$$
\Phi(z, s, a)=\sum_{i=0}^{\infty} \frac{z^{i}}{(a+i)^{s}},\left(a \in \mathbb{C} \backslash \mathbb{Z}_{0}^{-} ; s \in \mathbb{C} \text { when }|z|<1 ; \mathfrak{R}(s)>1 \text { when }|z|=1\right) \text {. }
$$

Recently many researchers investigated and studied various generalizations of the Hurwitz-Lerch zeta function $\Phi(z, s, a)$. The latest development and properties of such 
generalizations is found in the recent work of various researchers (see e.g., $[2,3,4,5,6$, $7,14,16,18,19,24,25])$. Very recently, Pathan and Daman [16] introduced another generalization in terms of double series representation:

$$
\phi_{\alpha, \beta ; \gamma: \lambda, \mu ; v}^{p, q}(z, t, s, a)=\sum_{i, j=0}^{\infty} \frac{(\alpha)_{i}(\beta)_{i}(\lambda)_{j}(\mu)_{j}}{(\gamma)_{i}(v)_{j}(a+p i+q j)^{s}} \frac{z^{i}}{i !} \frac{t^{j}}{j !}
$$

Where $\gamma, v, a \neq\{0,-1,-2, \ldots\}, \quad s \in \mathbb{C}, \quad p, q>0, \mathfrak{R}(s)>0$ when $|z|,|t|<1$ and $\mathfrak{R}(\gamma+\nu+s-\alpha-\beta-\lambda-\mu)>0$ when $|z|,|t|=1$.

Motivated essentially by various extensions of the Hurwitz-Lerch zeta function, we introduce a new extension of the generalized Hurwitz-Lerch zeta function of two variables defined as follows:

$$
\begin{aligned}
& p, q \\
& \Phi_{h: k ; l ; m, n: r ; v ; w}^{\alpha: \beta ; \gamma ; \lambda, \mu: v ; \rho ; \sigma} \\
&=\sum_{i, j=0}^{\infty} \frac{\left[\left(\alpha_{h}\right)\right]_{2 i+j}\left[\left(\beta_{k}\right)\right]_{i+j}\left[\left(\gamma_{l}\right)\right]_{i}\left[\left(\lambda_{m}\right)\right]_{j}}{\left[\left(\mu_{n}\right)\right]_{2 i+j}\left[\left(v_{r}\right)\right]_{i+j}\left[\left(\rho_{v}\right)\right]_{i}\left[\left(\sigma_{w}\right)\right]_{j}} \frac{z^{i} t^{j}}{i ! j !(a+p i+q j)^{s}},
\end{aligned}
$$

where $\alpha_{1}, \ldots, \alpha_{h}, \beta_{1}, \ldots, \beta_{k}, \gamma_{1}, \ldots, \gamma_{l}, \lambda_{1}, \ldots, \lambda_{m} \in \mathbb{C} ; \quad \mu_{1}, \ldots, \mu_{n}, v_{1}, \ldots, v_{r}, \rho_{1}, \ldots$ $\rho_{v}, \sigma_{1}, \ldots, \sigma_{w}, a \in \mathbb{C} \backslash \mathbb{Z}_{0}^{-} ; \quad p, q>0 ; \quad s \in \mathbb{C}, \quad \mathfrak{R}(s)>0 \quad$ when $\quad|z|,|t|<1$; $\quad$ and $\mathfrak{R}\left(\mu_{1}+\ldots+\mu_{n}+v_{1}+\ldots+v_{r}+\rho_{1}+\ldots+\rho_{v}+\sigma_{1}+\ldots+\sigma_{w}+s-\alpha_{1}-\ldots-\alpha_{h}-\beta_{1}-\right.$ $\left.\ldots-\beta_{k}-\gamma_{1}-\ldots-\gamma_{l}-\lambda_{1}-\ldots-\lambda_{m}\right)>0$ when $|z|,|t|=1$. Some special cases of (1.7) are given below.

In the case when $h=k=n=r=0, l=m=2$ and $v=w=1$, then (1.7) reduces to equation (11) of [16] which is given in (1.6). In the case when $k=r=0$, then we get

$$
{ }^{p, q} \Phi_{h: l ; m, n: v ; w}^{\alpha: \gamma ; \lambda, \mu: \rho ; \sigma}(z, t, s, a)=\sum_{i, j=0}^{\infty} \frac{\left[\left(\alpha_{h}\right)\right]_{2 i+j}\left[\left(\gamma_{l}\right)\right]_{i}\left[\left(\lambda_{m}\right)\right]_{j}}{\left[\left(\mu_{n}\right)\right]_{2 i+j}\left[\left(\rho_{v}\right)\right]_{i}\left[\left(\sigma_{w}\right)\right]_{j}} \frac{z^{i} t^{j}}{i ! j !(a+p i+q j)^{s}},
$$

where $\quad \alpha_{1}, \ldots, \alpha_{h}, \gamma_{1}, \ldots, \gamma_{l}, \lambda_{1}, \ldots, \lambda_{m} \in \mathbb{C} ; \quad \mu_{1}, \ldots, \mu_{n}, \rho_{1}, \ldots, \rho_{v}, \sigma_{1}, \ldots, \sigma_{w}, a \in$ $\mathbb{C} \backslash \mathbb{Z}_{0}^{-} ; \quad p, q>0 ; \quad s \in \mathbb{C}, \quad \mathfrak{R}(s)>0$ when $|z|,|t|<1$; and $\mathfrak{R}\left(\mu_{1}+\ldots+\mu_{n}+\rho_{1}+\right.$ $\left.\ldots+\rho_{v}+\sigma_{1}+\ldots+\sigma_{w}+s-\alpha_{1}-\ldots-\alpha_{h}-\gamma_{1}-\ldots-\gamma_{l}-\lambda_{1}-\ldots-\lambda_{m}\right)>0$ when $|z|$, $|t|=1$. Similarly, if $h=n=0$, we have 


$$
{ }^{p, q} \Phi_{k ; l ; m, r ; v ; w}^{\beta ; \gamma ; \lambda, v ; \rho ; \sigma}(z, t, s, a)=\sum_{i, j=0}^{\infty} \frac{\left[\left(\beta_{k}\right)\right]_{i+j}\left[\left(\gamma_{l}\right)\right]_{i}\left[\left(\lambda_{m}\right)\right]_{j}}{\left[\left(v_{r}\right)\right]_{i+j}\left[\left(\rho_{v}\right)\right]_{i}\left[\left(\sigma_{w}\right)\right]_{j}} \frac{z^{i} t^{j}}{i ! j !(a+p i+q j)^{s}}
$$

where $\quad \beta_{1}, \ldots, \beta_{k}, \gamma_{1}, \ldots, \gamma_{l}, \lambda_{1}, \ldots, \lambda_{m} \in \mathbb{C} ; \quad v_{1}, \ldots, v_{r}, \rho_{1}, \ldots, \rho_{v}, \sigma_{1}, \ldots, \sigma_{w}, a \in$ $\mathbb{C} \backslash \mathbb{Z}_{0}^{-} ; p, q>0 ; s \in \mathbb{C}, \mathfrak{R}(s)>0$ when $|z|,|t|<1$; and $\mathfrak{R}\left(\mathrm{v}_{1}+\ldots+\mathrm{v}_{r}+\rho_{1}+\ldots+\right.$ $\left.\rho_{v}+\sigma_{1}+\ldots+\sigma_{w}+s-\beta_{1}-\ldots-\beta_{k}-\gamma_{1}-\ldots-\gamma_{l}-\lambda_{1}-\ldots-\lambda_{m}\right)>0$ when $|z|,|t|$ $=1$, which, for $l=m=v=w=0$, we obtain the following:

$$
{ }^{p, q} \Phi_{k, r}^{\beta, v}(z, t, s, a)=\sum_{i, j=0}^{\infty} \frac{\left[\left(\beta_{k}\right)\right]_{i+j}}{\left[\left(v_{r}\right)\right]_{i+j}} \frac{z^{i} t^{j}}{i ! j !(a+p i+q j)^{s}},
$$

where $\beta_{1}, \ldots, \beta_{k} \in \mathbb{C} ; \quad v_{1}, \ldots, v_{r}, a \in \mathbb{C} \backslash \mathbb{Z}_{0}^{-} ; \quad p, q>0 ; \quad s \in \mathbb{C}, \quad \mathfrak{R}(s)>0$ when $|z|,|t|<1$; and $\mathfrak{R}\left(v_{1}+\ldots+v_{r}+s-\beta_{1}-\ldots-\beta_{k}\right)>0$ when $|z|,|t|=1$. Now, if in (1.10), we let $p=q=1$, then after some simplification, we have

$$
{ }^{1,1} \Phi_{k, r}^{\beta, v}(z, t, s, a)=\sum_{N=0}^{\infty} \frac{\left[\left(\beta_{k}\right)\right]_{N}}{\left[\left(v_{r}\right)\right]_{N}} \frac{(z+t)^{N}}{N !(a+N)^{s}},
$$

where $\beta_{1}, \ldots, \beta_{k} \in \mathbb{C} ; v_{1}, \ldots, v_{r}, a \in \mathbb{C} \backslash \mathbb{Z}_{0}^{-} ; s \in \mathbb{C}, \mathfrak{R}(s)>0$ when $|z|,|t|<1$; and $\mathfrak{R}\left(v_{1}+\ldots+v_{r}+s-\beta_{1}-\ldots-\beta_{k}\right)>0$ when $|z|,|t|=1$. By making suitable adjustments in the number of numerator and denominator parameters of (1.11), it gives the following relationships:

$$
\begin{gathered}
{ }^{1,1} \Phi_{1}^{\beta}(z, t, s, a)=\phi_{\beta}^{*}(z+t, s, a), \\
{ }^{1,1} \Phi_{1}^{1}(z, t, s, a)=\Phi(z+t, s, a), \\
{ }^{1,1} \Phi_{1}^{1}\left(\frac{1}{2}, \frac{1}{2}, s, a\right)=\zeta(s, a),
\end{gathered}
$$

where $\phi_{\beta}^{*}, \Phi$ and $\zeta(s, a)$ are the generalized Hurwitz-Lerch zeta function (see [11, p.100, (1.5)]), the Hurwitz-Lerch zeta function defined by (1.5) and the Hurwitz zeta function (see $[8$, p.24, (1)]), respectively. 


\section{Integral Representations}

Theorem 2.1. The following integral representation holds true for ${ }^{p, q} \Phi_{h: k ; l ; m, n: r ; v ; w}^{\alpha: \beta ; \lambda, \mu: v ; \rho ; \sigma}(z, t, s, a):$

$$
\begin{aligned}
& p, q \\
& \Phi_{h: k ; l ; m, n: r ; v ; w}^{\alpha: \beta ; \gamma ; \lambda, \mu ; v ; \rho ; \sigma}(z, t, s, a) \\
&=\frac{1}{\Gamma(s)} \int_{0}^{\infty} x^{s-1} e^{-a x} H_{n: r ; v ; w}^{h: k ; l ;}\left[\begin{array}{l}
\left(\alpha_{h}\right):\left(\beta_{k}\right) ;\left(\gamma_{l}\right) ;\left(\lambda_{m}\right) ; \\
\left(\mu_{n}\right):\left(v_{r}\right) ;\left(\rho_{v}\right) ;\left(\sigma_{w}\right) ;
\end{array} e^{-p x}, t e^{-q x}\right] d x,
\end{aligned}
$$

$(\min \{\mathfrak{R}(s), \mathfrak{R}(a)\}>0$ when $|z| \leq 1(z \neq 1), \quad|t| \leq 1(t \neq 1) ; \mathfrak{R}(s)>1$ when $z=1$, $t=1)$.

Proof. Using Eulerian integral formula (see, e.g., [8]):

$$
(a+i+j)^{-s}=\frac{1}{\Gamma(s)} \int_{0}^{\infty} x^{s-1} e^{-(a+i+j) x} d x, \quad\left(\min \{\mathfrak{R}(s), \mathfrak{R}(a)\}>0 ; i, j \in \mathbb{N}_{0}\right)
$$

in (1.7) and then, changing the order of the integral and the summation, and using definition (1.1), yields the proof of (2.1).

Similarly, by means of the relation (2.2), we can give the following corollary.

Corollary 2.1. The following integral representations for ${ }^{p, q} \Phi_{h: l ; m, n: v ; w}^{\alpha: \gamma ; \lambda, \mu: \rho ; \sigma}(z, t, s, a)$,

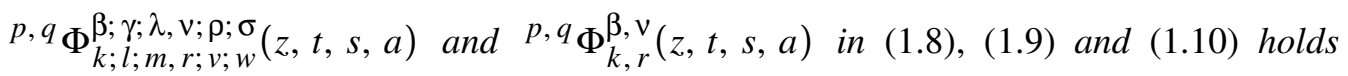
true:

$$
\begin{aligned}
& { }^{p, q} \Phi_{h: l ; m, n: v ; w}^{\alpha: \gamma ; \lambda, \mu: \rho ; \sigma}(z, t, s, a) \\
& =\frac{1}{\Gamma(s)} \int_{0}^{\infty} x^{s-1} e^{-a x} X_{n: v ; w}^{h: l ; m}\left[\begin{array}{l}
\left(\alpha_{h}\right):\left(\gamma_{l}\right) ;\left(\lambda_{m}\right) ; \\
\left(\mu_{n}\right):\left(\rho_{v}\right) ;\left(\sigma_{w}\right) ;
\end{array} e^{-p x}, t e^{-q x}\right] d x, \\
& { }^{p, q} \Phi_{k ; l ; m, r ; v ; w}^{\beta ; \gamma ; \lambda, v ; \rho ; \sigma}(z, t, s, a) \\
& =\frac{1}{\Gamma(s)} \int_{0}^{\infty} x^{s-1} e^{-a x} F_{r ; v ; w}^{k ; l ;}\left[\begin{array}{l}
\left(\beta_{k}\right) ;\left(\gamma_{l}\right) ;\left(\lambda_{m}\right) ; \\
\left(v_{r}\right) ;\left(\rho_{v}\right) ;\left(\sigma_{w}\right) ;
\end{array} e^{-p x}, t e^{-q x}\right] d x
\end{aligned}
$$


and

$$
{ }^{p, q} \Phi_{k, r}^{\beta, v}(z, t, s, a)=\frac{1}{\Gamma(s)} \int_{0}^{\infty} x^{s-1} e^{-a x}{ }_{k} F_{r}\left[\begin{array}{l}
\left(\beta_{k}\right) ; \\
\left(v_{r}\right) ;
\end{array} e^{-p x}+t e^{-q x}\right] d x
$$

$(\min \{\mathfrak{R}(s), \mathfrak{R}(a)\}>0$ when $|z| \leq 1(z \neq 1), \quad|t| \leq 1(t \neq 1) ; \mathfrak{R}(s)>1$ when $z=1$, $t=1)$.

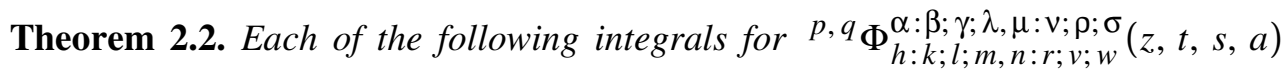
holds true when $h=n, k=r$ and $l=v$, respectively

$$
\begin{aligned}
& { }^{p, q} \Phi_{n: k ; l ; m, n: r ; v ; w}^{\alpha: \beta ; \gamma ; \lambda, \mu: v ; \rho ; \sigma}(z, t, s, a) \\
& =\frac{\Gamma\left(\mu_{1}\right) \cdots \Gamma\left(\mu_{n}\right)}{\Gamma\left(\alpha_{1}\right) \cdots \Gamma\left(\alpha_{n}\right) \Gamma\left(\mu_{1}-\alpha_{1}\right) \cdots \Gamma\left(\mu_{n}-\alpha_{n}\right)} \int_{0}^{1} \cdots \int_{0}^{1} x_{1}^{\alpha_{1}-1} \cdots x_{n}^{\alpha_{n}-1}\left(1-x_{1}\right)^{\mu_{1}-\alpha_{1}-1} \\
& \times \cdots\left(1-x_{1}\right)^{\mu_{n}-\alpha_{n}-1 p, q} \Phi_{k ; l ; m, r ; v ; w}^{\beta ; \gamma ; \lambda, v ; \rho ; \sigma}\left(x_{1}^{2} \cdots x_{n}^{2} z, x_{1} \cdots x_{n} t, s, a\right) d x_{1} \cdots d x_{n}, \\
& \left(\mathfrak{R}\left(\alpha_{u}\right)>0, \mathfrak{R}\left(\mu_{u}-\alpha_{u}\right)>0,(u=1,2, \ldots, n)\right),
\end{aligned}
$$

${ }^{p, q} \Phi_{h: k ; l ; m, n: k ; v ; w}^{\alpha: \beta ; \gamma, \mu, \sigma^{\prime}}(z, t, s, a)$

$=\frac{2^{k} \Gamma\left(v_{1}\right) \cdots \Gamma\left(v_{k}\right)}{\Gamma\left(\beta_{1}\right) \cdots \Gamma\left(\beta_{k}\right) \Gamma\left(v_{1}-\beta_{1}\right) \cdots \Gamma\left(v_{k}-\beta_{k}\right)} \int_{0}^{\frac{\pi}{2}} \cdots \int_{0}^{\frac{\pi}{2}}\left(\sin ^{2} x_{1}\right)^{\beta_{1}-\frac{1}{2}}\left(\cos ^{2} x_{1}\right)^{v_{1}-\beta_{1}-\frac{1}{2}}$

$$
\times \cdots\left(\sin ^{2} x_{k}\right)^{\beta_{k}-\frac{1}{2}}\left(\cos ^{2} x_{k}\right)^{v_{k}-\beta_{k}-\frac{1}{2} p, q_{\Phi^{2}}^{\alpha: \gamma ; \lambda, \mu: \rho ; \sigma ; m, n: v ; w}}\left(\sin ^{2} x_{1} \cdots \sin ^{2} x_{k} z,\right.
$$$$
\left.\sin ^{2} x_{1} \cdots \sin ^{2} x_{k} t, s, a\right) d x_{1} \cdots d x_{k},
$$$$
\left(\mathfrak{R}\left(\beta_{u}\right)>0, \mathfrak{R}\left(v_{u}-\beta_{u}\right)>0,(u=1,2, \ldots, k)\right),
$$

${ }^{p, q} \Phi_{h: k ; l ; m, n: r ; l ; w}^{\alpha: \beta ; \gamma ; \lambda, \mu: v ; \rho ; \sigma}(z, t, s, a)$

$$
=\frac{\Gamma\left(\rho_{1}\right) \cdots \Gamma\left(\rho_{l}\right)}{\Gamma\left(\gamma_{1}\right) \cdots \Gamma\left(\gamma_{l}\right) \Gamma\left(\rho_{1}-\gamma_{1}\right) \cdots \Gamma\left(\rho_{l}-\gamma_{l}\right)} \int_{0}^{\infty} \cdots \int_{0}^{\infty} \frac{x_{1}^{\gamma_{1}-1}}{\left(1+x_{1}\right)^{\rho_{1}}} \cdots \frac{x_{l}^{\gamma_{l}-1}}{\left(1+x_{l}\right)^{\rho_{l}}}
$$




$$
\begin{array}{r}
\times^{p, q} \Phi_{\substack{\alpha: \beta ; \lambda, \mu: v ; \sigma \\
h: k ; r ; w}}^{\alpha}\left(\frac{x_{1}}{\left(1+x_{1}\right)} \cdots \frac{x_{l}}{\left(1+x_{l}\right)} z, t, s, a\right) d x_{1} \cdots d x_{l}, \\
\left(\mathfrak{R}\left(\gamma_{u}\right)>0, \mathfrak{R}\left(\rho_{u}-\gamma_{u}\right)>0,(u=1,2, \ldots, l)\right) .
\end{array}
$$

Proof. To prove each of the integral representations from (2.6) to (2.8), it is enough to substitute the expression of the generalized Hurwitz-Lerch zeta function in each integrand and then to change the order of the integral and the summation, and finally taking into account the following Eulerian integrals (see, e.g., [8, p. 9-11], [19, Section $1.1]$ :

$$
\begin{gathered}
B(a, b)=\int_{0}^{1} x^{a-1}(1-x)^{b-1} d x \\
B(a, b)=2 \int_{0}^{\frac{\pi}{2}}(\sin x)^{2 a-1}(\cos x)^{2 b-1} d x \\
B(a, b)=\int_{0}^{\infty} \frac{x^{a-1}}{(1+x)^{a+b}} d x \\
(\mathfrak{R}(a)>0, \mathfrak{R}(b)>0) .
\end{gathered}
$$

Theorem 2.3. The following integral representations holds true:

$$
\begin{aligned}
& { }^{p, q} \Phi_{n: k ; l ; m, n: r ; v ; w}^{\alpha: \beta ; \gamma ; \lambda, \mu: v ; \rho ; \sigma}(z, t, s, a) \\
& =\frac{\Gamma\left(\mu_{1}\right) \cdots \Gamma\left(\mu_{n}\right)}{\Gamma\left(\alpha_{1}\right) \cdots \Gamma\left(\alpha_{n}\right) \Gamma\left(\mu_{1}-\alpha_{1}\right) \cdots \Gamma\left(\mu_{n}-\alpha_{n}\right) \Gamma(s)} \int_{0}^{\infty} \cdots \int_{0}^{\infty} e^{-\left(\alpha_{1} x_{1}+\cdots+\alpha_{n} x_{n}+a y\right)} \\
& \times\left(1-e^{-x_{1}}\right)^{\mu_{1-\alpha_{1}-1}} \cdots\left(1-e^{-x_{n}}\right)^{\mu_{n}-\alpha_{n}-1} y^{s-1} \\
& \times F_{r ; v ; w}^{k ; l ; m}\left[\begin{array}{l}
\left(\beta_{k}\right) ;\left(\gamma_{l}\right) ;\left(\lambda_{m}\right) ; \\
\left(v_{r}\right) ;\left(\rho_{v}\right) ;\left(\sigma_{w}\right) ;
\end{array} ; e^{-\left(2 x_{1}+\cdots+2 x_{n}+p y\right)}, t e^{-\left(x_{1}+\cdots+x_{n}+q y\right)}\right] d x_{1} \cdots d x_{n} d y, \\
& \left(\min \{\mathfrak{R}(s), \mathfrak{R}(a)\}>0 ; \mathfrak{R}\left(\alpha_{u}\right)>0, \mathfrak{R}\left(\mu_{u}-\alpha_{u}\right)>0,(u=1,2, \ldots, n)\right), \\
& { }^{p, q} \Phi_{h: k ; l ; m, n: k ; v ; w}^{\alpha: \beta ; \lambda, \mu: v ; \rho ; \sigma}(z, t, s, a)
\end{aligned}
$$




$$
\begin{aligned}
& =\frac{\Gamma\left(v_{1}\right) \cdots \Gamma\left(v_{k}\right)}{\Gamma\left(\beta_{1}\right) \cdots \Gamma\left(\beta_{k}\right) \Gamma\left(v_{1}-\beta_{1}\right) \cdots \Gamma\left(v_{k}-\beta_{k}\right) \Gamma(s)} \int_{0}^{\infty} \cdots \int_{0}^{\infty} \frac{x_{1}^{\beta_{1}-1} \cdots x_{k}^{\beta_{k}-1} y^{s-1} e^{-a y}}{\left(1+x_{1}\right)^{v_{1}} \cdots\left(1+x_{k}\right)^{v_{k}}}
\end{aligned}
$$

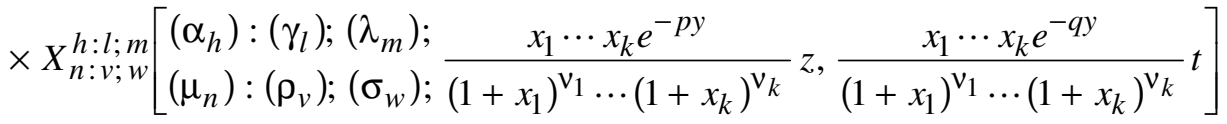

$$
\begin{aligned}
& \times d x_{1} \cdots d x_{k} d y, \\
& \left(\min \{\mathfrak{R}(s), \mathfrak{R}(a)\}>0 ; \mathfrak{R}\left(\beta_{u}\right)>0, \mathfrak{R}\left(v_{u}-\beta_{u}\right)>0,(u=1,2, \ldots, k)\right) .
\end{aligned}
$$

Proof. Using (2.2) and the Eulerian Beta function formula [8, p.11 (24)]

$$
B(a, b)=\int_{0}^{\infty}\left(e^{-x}\right)^{a}\left(1-e^{-x}\right)^{b-1} d x, \quad(\mathfrak{R}(a)>0, \mathfrak{R}(b)>0),
$$

which implies that

$$
\begin{aligned}
& { }^{p, q} \Phi_{\substack{\alpha: \beta ; \gamma ; \lambda, \mu: v ; \rho ; \sigma ; m, n: r ; v ; w \\
n: k ; j}}(z, t, s, a) \\
= & \frac{\Gamma\left(\mu_{1}\right) \cdots \Gamma\left(\mu_{n}\right)}{\Gamma\left(\alpha_{1}\right) \cdots \Gamma\left(\alpha_{n}\right) \Gamma\left(\mu_{1}-\alpha_{1}\right) \cdots \Gamma\left(\mu_{n}-\alpha_{n}\right) \Gamma(s)} \int_{0}^{\infty} \cdots \int_{0}^{\infty}\left(1-e^{-x_{1}}\right)^{\mu_{1}-\alpha_{1}-1} \\
& \times \cdots\left(1-e^{-x_{n}}\right)^{\mu_{n}-\alpha_{n}-1} e^{-\alpha_{1} x_{1}} \cdots e^{-\alpha_{n} x_{n}} y^{s-1} e^{-a y} \\
& \times \sum_{i, j=0}^{\infty} \frac{\left[\left(\beta_{k}\right)\right]_{i+j}\left[\left(\gamma_{l}\right)\right]_{i}\left[\left(\lambda_{m}\right)\right]_{j}}{\left[\left(v_{r}\right)\right]_{i+j}\left[\left(\rho_{v}\right)\right]_{i}\left[\left(\sigma_{w}\right)\right]_{j}} \frac{\left(z e^{-\left(2 x_{1}+\cdots+2 x_{n}+p y\right)}\right)^{i}\left(t e^{-\left(x_{1}+\cdots+x_{n}+q y\right)}\right)^{j}}{i ! j !} \\
& \times d x_{1} \cdots d x_{n} d y .
\end{aligned}
$$

in view of definition (1.2), immediately yields the first equality (2.12). By employing formulas (2.2) and (2.11), and exploiting the same procedure leading to (2.12) one can derive the second equality (2.13).

Now, by using the contour integral [8, p.14 (4)]

$$
\Gamma(s)=\frac{-1}{2 i \sin (\pi s)} \int_{\infty}^{(0+)}(-x)^{s-1} e^{-x} d x,|\arg (-x)| \leq \pi,
$$

we can give the following theorem without proof. 
Theorem 2.4. Let $\mathfrak{R}(a)>0$ and $|\arg (-x)| \leq \pi$. Then

$$
\begin{aligned}
& p, q \Phi_{h: k ; l ; m, n: r ; v ; w}^{\alpha: \beta ; \gamma, \mu: v ; \sigma ; \sigma}(z, t, s, a) \\
= & \frac{-\Gamma(1-s)}{2 \pi i} \int_{\infty}^{(0+)}(-x)^{s-1} e^{-a x} H_{n: r ; v ; w}^{h: k ; l ; m}\left[\begin{array}{l}
\left(\alpha_{h}\right):\left(\beta_{k}\right) ;\left(\gamma_{l}\right) ;\left(\lambda_{m}\right) ; \\
\left(\mu_{n}\right):\left(v_{r}\right) ;\left(\rho_{v}\right) ;\left(\sigma_{w}\right) ;
\end{array} e^{-p x}, t e^{-q x}\right] d x .
\end{aligned}
$$

Theorem 2.5. The following Mellin-Barnes contour integral representation of (1.7) holds true:

$$
\begin{aligned}
&{ }^{p, q} \Phi_{h: k ; l ; m, n: r ; v ; w}^{\alpha: \beta ; \gamma ; \lambda ;}(z, t, s, a) \\
&=-\frac{\prod_{u=1}^{n} \Gamma\left(\mu_{u}\right) \prod_{u=1}^{r} \Gamma\left(v_{u}\right) \prod_{u=1}^{v} \Gamma\left(\rho_{u}\right) \prod_{u=1}^{w} \Gamma\left(\sigma_{u}\right)}{4 \pi^{2} \prod_{u=1}^{h} \Gamma\left(\alpha_{u}\right) \prod_{u=1}^{k} \Gamma\left(\beta_{u}\right) \prod_{u=1}^{l} \Gamma\left(\gamma_{u}\right) \prod_{u=1}^{m} \Gamma\left(\lambda_{u}\right)} \int_{C_{z}} \int_{C_{t}} \\
& \times \frac{\Gamma(-x) \Gamma(-y) \prod_{u=1}^{h} \Gamma\left(\alpha_{u}+2 x+y\right) \prod_{u=1}^{k} \Gamma\left(\beta_{u}+x+y\right) \prod_{u=1}^{l} \Gamma\left(\gamma_{u}+x\right) \prod_{u=1}^{m} \Gamma\left(\lambda_{u}+y\right)}{\prod_{u=1}^{n} \Gamma\left(\mu_{u}+2 x+y\right) \prod_{u=1}^{r} \Gamma\left(v_{u}+x+y\right) \prod_{u=1}^{v} \Gamma\left(\rho_{u}+x\right) \prod_{u=1}^{w} \Gamma\left(\sigma_{u}+y\right)} \\
& \times \frac{[\Gamma(a+p x+q y)]^{s}(-z)^{x}(-t)^{y}}{[\Gamma(a+p x+q y+1)]^{s}} d x d y, \\
&(|\arg (-z)|<\pi,|\arg (-t)|<\pi) .
\end{aligned}
$$

Proof. If we evaluate the integral as a sum of the residues by calculus of residues at the simple poles of $\Gamma(-x)$ at the points $x=-i\left(i \in \mathbb{N}_{0}\right)$ and $\Gamma(-y)$ at the points $y=-j\left(j \in \mathbb{N}_{0}\right)$, we immediately find the following series expansion:

$$
\frac{\prod_{u=1}^{n} \Gamma\left(\mu_{u}\right) \prod_{u=1}^{r} \Gamma\left(v_{u}\right) \prod_{u=1}^{v} \Gamma\left(\rho_{u}\right) \prod_{u=1}^{w} \Gamma\left(\sigma_{u}\right)}{\prod_{u=1}^{h} \Gamma\left(\alpha_{u}\right) \prod_{u=1}^{k} \Gamma\left(\beta_{u}\right) \prod_{u=1}^{l} \Gamma\left(\gamma_{u}\right) \prod_{u=1}^{m} \Gamma\left(\lambda_{u}\right)}
$$




$$
\begin{aligned}
& \times \sum_{i, j=0}^{\infty} \frac{\prod_{u=1}^{h} \Gamma\left(\alpha_{u}+2 i+j\right) \prod_{u=1}^{k} \Gamma\left(\beta_{u}+i+j\right) \prod_{u=1}^{l} \Gamma\left(\gamma_{u}+i\right) \prod_{u=1}^{m} \Gamma\left(\lambda_{u}+j\right)}{\prod_{u=1}^{n} \Gamma\left(\mu_{u}+2 i+j\right) \prod_{u=1}^{r} \Gamma\left(v_{u}+i+j\right) \prod_{u=1}^{v} \Gamma\left(\rho_{u}+i\right) \prod_{u=1}^{w} \Gamma\left(\sigma_{u}+j\right)} \\
& \times \frac{z^{i} t^{j}}{i ! j !(a+p i+q j)^{s}},
\end{aligned}
$$

which is just the ${ }^{p, q} \Phi_{h: k ; l ; m, n: r ; v ; w}^{\alpha: \beta ; \gamma ; \mu, \nu ; \rho ; \sigma}(z, t, s, a)$. This completes the proof.

By exploiting the following well-known integral formula [12]:

$$
\begin{gathered}
2 \pi i(1-\eta)^{-s} \Gamma(s)=\int_{c-i \infty}^{c+i \infty} \Gamma(s+x) \Gamma(-x)(-\eta)^{x} d x, \\
(\eta, s \in \mathbb{C}, \mathfrak{R}(s)>0 ;|\arg (\eta)|<\pi),
\end{gathered}
$$

we can derive the following integral representation.

Theorem 2.6. For $\mathfrak{R}(b)>0, \mathfrak{R}(a-b)>0$ and $\mathfrak{R}(s)>0$, the following integral holds true:

$$
\begin{aligned}
& { }^{p, q} \Phi_{h: k ; l ; m, n: r ; v ; w}^{\alpha: \beta ; \gamma ; \lambda, \mu: v ; \rho ; \sigma}(z, t, s, a)
\end{aligned}
$$

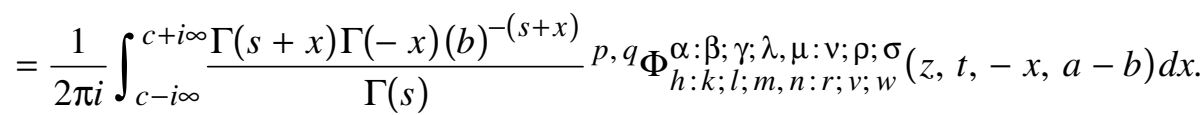

Proof. Let us denote, for convenience, the left-hand side of assertion (2.18) by $\Lambda$. Then considering the definition (1.7), it is easily seen that:

$$
\begin{aligned}
\Lambda= & \sum_{i, j=0}^{\infty} \frac{\left[\left(\alpha_{h}\right)\right]_{2 i+j}\left[\left(\beta_{k}\right)\right]_{i+j}\left[\left(\gamma_{l}\right)\right]_{i}\left[\left(\lambda_{m}\right)\right]_{j}}{\left[\left(\mu_{n}\right)\right]_{2 i+j}\left[\left(v_{r}\right)\right]_{i+j}\left[\left(\rho_{v}\right)\right]_{i}\left[\left(\sigma_{w}\right)\right]_{j}} \frac{z^{i} t^{j}}{i ! j !} \\
& \times(b)^{-s}\left[\frac{1}{2 \pi i} \int_{c-i \infty}^{c+i \infty} \frac{\Gamma(s+x) \Gamma(-x)}{\Gamma(s)}\left(\frac{a-b+p i+q j}{b}\right)^{x} d x\right],
\end{aligned}
$$

which, upon using (2.17), leads to the desired formula in (2.18). 


\section{Operational Connections}

Here we begin by recalling the following formula is known consequences of the derivative operator $D_{x}[15]$ :

$$
D_{x}^{u} x^{\eta}=\frac{\Gamma(\eta+1)}{\Gamma(\eta-u+1)} x^{\eta-u}, \quad \eta-u \geq 0 .
$$

Theorem 3.1. The following results hold true:

$$
\begin{aligned}
& D_{z}^{u p, q} \Phi_{h: k ; l ; m, n: r ; v ; w}^{\alpha: \beta ; \gamma ; \lambda, \mu: v ; \rho ; \sigma}(z, t, s, a) \\
& =\frac{\left[\left(\alpha_{h}\right)\right]_{2 u}\left[\left(\beta_{k}\right)\right]_{u}\left[\left(\gamma_{l}\right)\right]_{u}}{\left[\left(\mu_{n}\right)\right]_{2 u}\left[\left(v_{r}\right)\right]_{u}\left[\left(\rho_{v}\right)\right]_{u}} p, q \Phi_{h: k ; l ; m, n: r ; v ; w}^{\alpha+2 u: \beta+u ; \gamma+u ; \mu+2 u: v+u ; \rho+u ; \sigma}(z, t, s, a+p u), \\
& D_{t}^{u p, q} \Phi_{h: k ; l ; m, n: r ; v ; w}^{\alpha: \beta ; \gamma ; \lambda, \mu: v ; \rho ; \sigma}(z, t, s, a) \\
& =\frac{\left[\left(\alpha_{h}\right)\right]_{u}\left[\left(\beta_{k}\right)\right]_{u}\left[\left(\lambda_{l}\right)\right]_{u}}{\left[\left(\mu_{n}\right)\right]_{u}\left[\left(v_{r}\right)\right]_{u}\left[\left(\sigma_{v}\right)\right]_{u}} p, q \Phi_{h: k ; l ; m, n: r ; v ; w}^{\alpha+u: \beta+u ; \gamma, \lambda+u, \mu+u: v+u ; \rho ; \sigma+u}(z, t, s, a+q u), \\
& D_{z}^{u} D_{t}^{u^{\prime} p, q} \Phi_{h: k ; l ; m, n: r ; v ; w}^{\alpha: \beta ; \gamma ; \lambda, \mu ;}(z, t, s, a) \\
& =\frac{\left[\left(\alpha_{h}\right)\right]_{2 u+u^{\prime}}\left[\left(\beta_{k}\right)\right]_{u+u^{\prime}}\left[\left(\gamma_{l}\right)\right]_{u}\left[\left(\lambda_{l}\right)\right]_{u^{\prime}}}{\left[\left(\mu_{n}\right)\right]_{2 u+u^{\prime}}\left[\left(v_{r}\right)\right]_{u+u^{\prime}}\left[\left(\rho_{v}\right)\right]_{u}\left[\left(\sigma_{v}\right)\right]_{u^{\prime}}} \\
& \times{ }^{p, q} \Phi_{h: k ; l ; m, n: r ; v ; w}^{\alpha+2 u+u^{\prime}: \beta+u+u^{\prime} ; \gamma+u ; \lambda+u^{\prime}, \mu+2 u+u^{\prime}: v+u+u^{\prime} ; \rho+u ; \sigma+u^{\prime}}\left(z, t, s, a+p u+q u^{\prime}\right), \\
& D_{a}^{u p, q} \Phi_{h: k ; l ; m, n: r ; v ; w}^{\alpha: \beta ; \gamma ; \lambda, \mu: v ; \rho ; \sigma}(z, t, s, a) \\
& =(-1)^{u}(s)_{u}^{p, q} \Phi_{h: k ; l ; m, n: r ; v ; w}^{\alpha: \beta ; \gamma, \mu: v ; \beta}(z, t, s+u, a), \quad(u \in \mathbb{N}) .
\end{aligned}
$$

Proof. L.H.S of (3.2) after using (3.1) gives

$$
D_{z}^{u p, q} \Phi_{h: k ; l ; m, n: r ; v ; w}^{\alpha: \beta ; \gamma ; \lambda, \mu: v ; \rho ; \sigma}(z, t, s, a)
$$




$$
=\sum_{i, j=0}^{\infty} \frac{\left[\left(\alpha_{h}\right)\right]_{2 i+j}\left[\left(\beta_{k}\right)\right]_{i+j}\left[\left(\gamma_{l}\right)\right]_{i}\left[\left(\lambda_{m}\right)\right]_{j}}{\left[\left(\mu_{n}\right)\right]_{2 i+j}\left[\left(v_{r}\right)\right]_{i+j}\left[\left(\rho_{v}\right)\right]_{i}\left[\left(\sigma_{w}\right)\right]_{j}} \frac{z^{i-u} t^{j}}{(i-u) ! j !(a+p i+q j)^{s}} .
$$

If we put $i \rightarrow i+u$ in (3.6), after little simplification and using definition (1.7), yields the result (3.2). In the same manner, one can prove the relations (3.3)-(3.5).

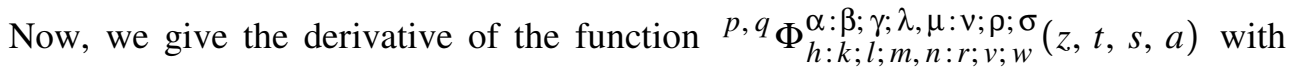
respect to the argument $p$ and $q$, respectively.

Theorem 3.2. Let $b \in \mathbb{R}$. Then

$$
\begin{aligned}
& \frac{\partial}{\partial p} p, q \Phi_{h: k ; l ; m, n: r ; v ; w}^{\alpha: \beta ; \gamma ; \lambda, \mu: v ; \rho ; \sigma}(z, t, s-1, a+p b) \\
& =(1-s)\left[\frac{\left[\left(\alpha_{h}\right)\right]_{2}\left[\left(\beta_{k}\right)\right]\left[\left(\gamma_{l}\right)\right]}{\left[\left(\mu_{n}\right)\right]_{2}\left[\left(v_{r}\right)\right]\left[\left(\rho_{v}\right)\right]} z^{p, q} \Phi_{h: k ; l ; m, n: r ; v ; w}^{\alpha+2 ; \beta+1 ; \gamma+1 ; \lambda, \mu+2: v+1 ; \rho+1 ; \sigma}(z, t, s, a+p(b+1))\right. \\
& \left.+b^{p, q} \Phi_{h: k ; l ; m, n: r ; v ; w}^{\alpha: \beta ; \gamma ; \lambda, \mu: v ; \rho ; \sigma}(z, t, s, a+p b)\right], \\
& \frac{\partial}{\partial q} p, q \Phi_{h: k ; l ; m, n: r ; v ; w}^{\alpha: \beta ; \gamma ; \lambda, \mu: v ; \beta ; \sigma}(z, t, s-1, a+q b) \\
& =(1-s)\left[\frac{\left[\left(\alpha_{h}\right)\right]\left[\left(\beta_{k}\right)\right]\left[\left(\lambda_{l}\right)\right]}{\left[\left(\mu_{n}\right)\right]\left[\left(v_{r}\right)\right]\left[\left(\sigma_{v}\right)\right]} t^{p, q} \Phi_{h: k ; l ; m, n: r ; v ; w}^{\alpha+1: \beta+1 ; \gamma ; \lambda+1, \mu+1: v+1 ; \rho ; \sigma+1}(z, t, s, a+q(b+1))\right. \\
& +b^{p, q} \Phi_{h: k ; l ; m, n: r ; v ; w}^{\alpha: \beta ; \gamma ; \lambda, \mu ;:, i, s, a+q b)]} \text {. }
\end{aligned}
$$

Proof. We have

$$
\begin{aligned}
& \frac{\partial}{\partial p} p, q \Phi_{h: k ; l ; m, n: r ; v ; w}^{\alpha: \beta ; \gamma ; \lambda, \mu: v ; \rho ; \sigma}(z, t, s-1, a+p b) \\
& =(1-s)\left[\sum_{i=1}^{\infty} \sum_{j=0}^{\infty} \frac{\left[\left(\alpha_{h}\right)\right]_{2 i+j}\left[\left(\beta_{k}\right)\right]_{i+j}\left[\left(\gamma_{l}\right)\right]_{i}\left[\left(\lambda_{m}\right)\right]_{j}}{\left[\left(\mu_{n}\right)\right]_{2 i+j}\left[\left(v_{r}\right)\right]_{i+j}\left[\left(\rho_{v}\right)\right]_{i}\left[\left(\sigma_{w}\right)\right]_{j}} \frac{z^{i} t^{j}}{(i-1) ! j !(a+p(b+i)+q j)^{s}}\right.
\end{aligned}
$$




$$
\left.b \sum_{i=0}^{\infty} \sum_{j=0}^{\infty} \frac{\left[\left(\alpha_{h}\right)\right]_{2 i+j}\left[\left(\beta_{k}\right)\right]_{i+j}\left[\left(\gamma_{l}\right)\right]_{i}\left[\left(\lambda_{m}\right)\right]_{j}}{\left[\left(\mu_{n}\right)\right]_{2 i+j}\left[\left(v_{r}\right)\right]_{i+j}\left[\left(\rho_{v}\right)\right]_{i}\left[\left(\sigma_{w}\right)\right]_{j}} \frac{z^{i} t^{j}}{i ! j !(a+p(b+i)+q j)^{s}}\right] .
$$

By setting $i \rightarrow i+1$ in the first summation of the equality (3.9), and simplifying, we obtain the result (3.7). Similarly, one can prove the equality (3.8). In similar way, we can prove the following theorem.

Theorem 3.3. Let $u \in \mathbb{R}$. Then

$$
\begin{aligned}
& \frac{\partial}{\partial u} p, q \Phi_{h: k ; l ; m, n: r ; v ; w}^{\alpha: \beta ; \gamma ; \lambda, \mu: v ; \rho ; \sigma}(z, t, s-1, a+u b) \\
& =b(1-s)^{p, q} \Phi_{h: k ; l ; m, n: r ; v ; w}^{\alpha: \beta ; \gamma ; \lambda, \mu: v ; \rho ; \sigma}(z, t, s, a+u b) .
\end{aligned}
$$

On other hand side, we now use the following equality in order to derive the differentiation formula for our generalized Hurwitz-Lerch zeta function of two variables:

$$
(a+p i+q j)^{-s}=e^{-s \log (a+p i+q j)} .
$$

\section{Theorem 3.4.}

$$
\begin{aligned}
& D_{s}^{u} p, q \Phi_{h: k ; l ; m, n: r ; v ; w}^{\alpha: \beta ; \gamma ; \lambda, \mu: v ; \beta ; \sigma}(z, t, s, a) \\
= & \sum_{i, j=0}^{\infty} \frac{\left[\left(\alpha_{h}\right)\right]_{2 i+j}\left[\left(\beta_{k}\right)\right]_{i+j}\left[\left(\gamma_{l}\right)\right]_{i}\left[\left(\lambda_{m}\right)\right]_{j}}{\left[\left(\mu_{n}\right)\right]_{2 i+j}\left[\left(v_{r}\right)\right]_{i+j}\left[\left(\rho_{v}\right)\right]_{i}\left[\left(\sigma_{w}\right)\right]_{j}} \frac{z^{i} t^{j}[-\log (a+p i+q j)]^{u}}{i ! j !(a+p i+q j)^{s}}, u>0 .
\end{aligned}
$$

Proof. By using the formula (3.11) in (1.7) and differentiating with respect to $s$, we can easily obtain the desired result.

Further, if we use the diagamma function, defined by [1, p.74 (2.51)]

$$
\psi(y)=\frac{d}{d y} \ln \Gamma(y)=\frac{\Gamma^{\prime}(y)}{\Gamma(y)}, \quad y \neq 0,-1,-2, \ldots
$$

other differentiation formula would occur as follows:

Theorem 3.5. For the generalized Hurwitz-Lerch zeta function (1.7), we have

$$
\prod_{u=1}^{h}\left(\frac{\partial}{\partial \alpha_{u}}\right) p, q \Phi_{h: k ; l ; m, n: r ; v ; w}^{\alpha: \beta ; \gamma ; \lambda, \mu: v ; \rho ; \sigma}(z, t, s, a)
$$




$$
\begin{aligned}
& =\sum_{i, j=0}^{\infty} \frac{\left[\left(\alpha_{h}\right)\right]_{2 i+j}\left[\left(\beta_{k}\right)\right]_{i+j}\left[\left(\gamma_{l}\right)\right]_{i}\left[\left(\lambda_{m}\right)\right]_{j}}{\left[\left(\mu_{n}\right)\right]_{2 i+j}\left[\left(v_{r}\right)\right]_{i+j}\left[\left(\rho_{v}\right)\right]_{i}\left[\left(\sigma_{w}\right)\right]_{j}} \frac{z^{i} t^{j}}{i ! j !(a+p i+q j)^{s}} \\
& \prod_{u=1}^{h}\left[\psi\left(\alpha_{u}+2 i+j\right)-\psi\left(\alpha_{u}\right)\right] \\
& \left(\alpha_{u} \in \mathbb{C} \backslash \mathbb{Z}_{0}^{-}, u=1,2, \ldots, h\right) .
\end{aligned}
$$

Proof. Equation (3.14) can be easily obtained by using (3.13). Finally, we derive the following connections.

Theorem 3.6. If $\mathfrak{R}\left(\gamma_{l}\right)>0$ and $\mathfrak{R}\left(\lambda_{m}\right)>0$, then following results hold true:

$$
\begin{aligned}
& D_{z}^{\left(\gamma_{l}-\rho_{v}\right)}\left[z^{\gamma_{l}-1 p, q} \Phi_{h: k ; l-1 ; m, n: r ; v-1 ; w}^{\alpha: \beta ; \gamma ; \lambda, \mu: v ; \rho ; \sigma}(z, t, s, a)\right] \\
& =\frac{\Gamma\left(\gamma_{l}\right)}{\Gamma\left(\rho_{v}\right)} z^{\rho_{v}-1 p, q} \Phi_{h: k ; l ; m, n: r ; v ; w}^{\alpha: \beta ; \gamma ; \lambda, \mu ; v ; \rho ; \sigma}(z, t, s, a), \\
& D_{z}^{\left(\gamma_{l}-\rho_{v}\right)} D_{t}^{\left(\lambda_{m}-\sigma_{w}\right)}\left[z^{\gamma_{l}-1} t^{\lambda_{m}-1} p, q \Phi_{h: k ; l-1 ; m-1, n: r ; v-1 ; w-1}^{\alpha: \beta ; \gamma ; \lambda, \mu: v ; \rho ; \sigma}(z, t, s, a)\right]
\end{aligned}
$$

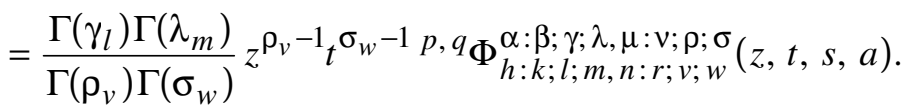

Proof. Consider the left hand side of (3.15), then in view of (1.7), we have

$$
\begin{aligned}
& D_{z}^{\left(\gamma_{l}-\rho_{v}\right)}\left[z^{\gamma_{l}-1 p, q} \Phi_{h: k ; l-1 ; m, n: r ; v-1 ; w}^{\alpha: \beta ; \gamma, \lambda, \mu: v ; \rho ; \sigma}(z, t, s, a)\right] \\
& =D_{z}^{\left(\gamma_{l}-\rho_{v}\right)}\left[\sum_{i, j=0}^{\infty} \frac{\left[\left(\alpha_{h}\right)\right]_{2 i+j}\left[\left(\beta_{k}\right)\right]_{i+j}\left[\left(\gamma_{l-1}\right)\right]_{i}\left[\left(\lambda_{m}\right)\right]_{j}}{\left[\left(\mu_{n}\right)\right]_{2 i+j}\left[\left(\nu_{r}\right)\right]_{i+j}\left[\left(\rho_{v-1}\right)\right]_{i}\left[\left(\sigma_{w}\right)\right]_{j}} \frac{z^{\gamma_{l}+i-1} t^{j}}{i ! j !(a+p i+q j)^{s}}\right] .
\end{aligned}
$$

Upon using the relation (3.1) readily leads to the right hand side of (3.15). Applying the similar procedure used in (3.15), we get the formula (3.16).

\section{Summation Formulas}

In this section, we obtain some sums for the generalized Hurwitz-Lerch zeta function ${ }^{p, q} \Phi_{h: k ; l ; m, n: r ; v ; w}^{\alpha: \beta ; \gamma ; \lambda, \mu: v ; \beta ; \sigma}(z, t, s, a)$ as follows: 
Theorem 4.1. The following summation formula hold true:

$$
\begin{aligned}
& { }^{p, q} \Phi_{h: k ; l ; m, n: r ; v ; w}^{\alpha: \beta ; \gamma ; \lambda, \mu: v ; \rho ; \sigma}(z, t, s, a-x)=\sum_{u=0}^{\infty} \frac{(s)_{u}}{u !}{ }^{p, q} \Phi_{h: k ; l ; m, n: r ; v ; w}^{\alpha: \beta ; \gamma ; \lambda, \mu: v ; \rho ; \sigma}(z, t, s+u, a) x^{u}, \\
& (|x|<|a| ; s \neq 1) \text {. }
\end{aligned}
$$

Proof. Using (1.7), we get

$$
\begin{aligned}
& p, q \\
& \Phi_{h}^{\alpha: \beta ; \gamma ; \lambda ; \mu, \mu: v ; \rho ; \sigma ; \gamma ; w} \\
&= \sum_{i, j=0}^{\infty} \frac{\left[\left(\alpha_{h}\right)\right]_{2 i+j}\left[\left(\beta_{k}\right)\right]_{i+j}\left[\left(\gamma_{l}\right)\right]_{i}\left[\left(\lambda_{m}\right)\right]_{j}}{\left[\left(\mu_{n}\right)\right]_{2 i+j}\left[\left(v_{r}\right)\right]_{i+j}\left[\left(\rho_{v}\right)\right]_{i}\left[\left(\sigma_{w}\right)\right]_{j}} \frac{z^{i} t^{j}}{i ! j !(p i+q j+a-x)^{s}} \\
&= \sum_{i, j=0}^{\infty} \frac{\left[\left(\alpha_{h}\right)\right]_{2 i+j}\left[\left(\beta_{k}\right)\right]_{i+j}\left[\left(\gamma_{l}\right)\right]_{i}\left[\left(\lambda_{m}\right)\right]_{j}}{\left[\left(\mu_{n}\right)\right]_{2 i+j}\left[\left(v_{r}\right)\right]_{i+j}\left[\left(\rho_{v}\right)\right]_{i}\left[\left(\sigma_{w}\right)\right]_{j}} \frac{z^{i} t^{j}}{i ! j !(a+p i+q j)^{s}}\left(1-\frac{x}{a+p i+q j}\right)^{-s} \\
&= \sum_{u=0}^{\infty} \frac{(s)_{u}}{u !}\left[\sum_{i, j=0}^{\infty} \frac{\left[\left(\alpha_{h}\right)\right]_{2 i+j}\left[\left(\beta_{k}\right)\right]_{i+j}\left[\left(\gamma_{l}\right)\right]_{i}\left[\left(\lambda_{m}\right)\right]_{j}}{\left[\left(\mu_{n}\right)\right]_{2 i+j}\left[\left(v_{r}\right)\right]_{i+j}\left[\left(\rho_{v}\right)\right]_{i}\left[\left(\sigma_{w}\right)\right]_{j}} \frac{z^{i} t^{j}}{i ! j !(a+p i+q j)^{s+u}}\right] x^{u},
\end{aligned}
$$

which in view of definition (1.7), yields proof of (4.1).

Theorem 4.2. Let $\max \{|z / b|,|t / b|\}<1$ and $|b|<\mathfrak{R}(a)$. Then

$$
\begin{aligned}
& \sum_{u, u^{\prime}=0}^{\infty} \frac{(\eta)_{u}\left(\eta^{\prime}\right)_{u^{\prime}}}{u ! u^{\prime} !} p, q \Phi_{h: k ; \gamma ; m, n: v ; \rho ; \sigma ; w}^{\alpha: \sigma}\left(z, t, s+u+u^{\prime}, a-b\right) x^{u} y^{u^{\prime}} \\
= & \sum_{i, j=0}^{\infty} \frac{\left[\left(\alpha_{h}\right)\right]_{2 i+j}\left[\left(\beta_{k}\right)\right]_{i+j}\left[\left(\gamma_{l}\right)\right]_{i}\left[\left(\lambda_{m}\right)\right]_{j}}{\left[\left(\mu_{n}\right)\right]_{2 i+j}\left[\left(v_{r}\right)\right]_{i+j}\left[\left(\rho_{v}\right)\right]_{i}\left[\left(\sigma_{w}\right)\right]_{j}} \frac{z^{i} t^{j}}{i ! j !(a+p i+q j)^{s}} \\
& \times F_{G}\left(s, s, s, 1, \eta, \eta^{\prime} ; 1, s, s ; \frac{b}{(a+p i+q j)}, \frac{x}{(a+p i+q j)}, \frac{y}{(a+p i+q j)}\right) .
\end{aligned}
$$

Proof. We have

$$
\sum_{u, u^{\prime}=0}^{\infty} \frac{(\eta)_{u}\left(\eta^{\prime}\right)_{u^{\prime}} p, q}{u ! u^{\prime} !} \Phi_{h: k ; l ; m, n: r ; v ; w}^{\alpha: \beta ; \gamma ; \lambda, \mu: v ; \rho ; \sigma}\left(z, t, s+u+u^{\prime}, a-b\right) x^{u} y^{u^{\prime}}
$$




$$
\begin{aligned}
= & \sum_{i, j=0}^{\infty} \frac{\left[\left(\alpha_{h}\right)\right]_{2 i+j}\left[\left(\beta_{k}\right)\right]_{i+j}\left[\left(\gamma_{l}\right)\right]_{i}\left[\left(\lambda_{m}\right)\right]_{j}}{\left[\left(\mu_{n}\right)\right]_{2 i+j}\left[\left(v_{r}\right)\right]_{i+j}\left[\left(\rho_{v}\right)\right]_{i}\left[\left(\sigma_{w}\right)\right]_{j}} \frac{z^{i} t^{j}}{i ! j !(a+p i+q j)^{s}} \\
& \times \sum_{u, u^{\prime}, u^{\prime \prime}=0}^{\infty} \frac{(s)_{u+u^{\prime}+u^{\prime \prime}}(\eta)_{u}\left(\eta^{\prime}\right)_{u^{\prime}}}{(s)_{u+u^{\prime}} u ! u^{\prime} ! u^{\prime \prime} !}\left[\frac{b}{(a+p i+q j)}\right]^{u^{\prime \prime}}\left[\frac{x}{(a+p i+q j)}\right]^{u}\left[\frac{y}{(a+p i+q j)}\right]^{u^{\prime}} .
\end{aligned}
$$

Now, by using Lauricella's series of three variables $F_{G}$ defined by (see $\left.[13,20]\right)$

$$
\begin{aligned}
& F_{G}\left(\xi, \xi, \xi, \eta, \eta^{\prime}, \eta^{\prime \prime} ; \tau, v, v ; x_{1}, x_{2}, x_{3}\right) \\
= & \sum_{u, u^{\prime}, u^{\prime \prime}=0}^{\infty} \frac{(\xi)_{u+u^{\prime}+u^{\prime \prime}}(\eta)_{u}\left(\eta^{\prime}\right)_{u^{\prime}}\left(\eta^{\prime \prime}\right)_{u^{\prime \prime}}}{(\tau)_{u}(v)_{u^{\prime}+u^{\prime \prime}}} \frac{x_{1}^{u} x_{2}^{u^{\prime}} x_{3}^{u^{\prime \prime}}}{u ! u^{\prime} ! u^{\prime \prime} !}
\end{aligned}
$$

we get the required result.

Theorem 4.3. The following relation holds true:

$$
\begin{aligned}
& { }^{p, q} \Phi_{h: k ; l ; m, n: r ; v ; w}^{\alpha: \beta ; \gamma ; \lambda, \mu: v ; \rho ; \sigma}(z+x, t, s, a) \\
= & \sum_{u=0}^{\infty} \frac{\left[\left(\alpha_{h}\right)\right]_{u}\left[\left(\beta_{k}\right)\right]_{u}\left[\left(\lambda_{l}\right)\right]_{u}}{\left[\left(\mu_{n}\right)\right]_{u}\left[\left(v_{r}\right)\right]_{u}\left[\left(\sigma_{v}\right)\right]_{u}} \\
& \times{ }^{p, q} \Phi_{h: k ; l ; m, n: r ; v ; w}^{\alpha+u: \beta+u ; \gamma ; \lambda+u, \mu+u: v+u ; \rho ; \sigma+u}(z, t, s, a+q u) \frac{x^{u}}{u !}, \quad(|x|<1) .
\end{aligned}
$$

Proof. The proof is a direct application of the formula (cf. [17, p.63, (2.8.8)]

$$
f(x+y)=\sum_{u=0}^{\infty} f^{(u)}(x) \frac{y^{u}}{u !} .
$$

Theorem 4.4. The following summation formula for the generalized Hurwitz-Lerch zeta function (1.7) holds true for $s \neq 0,1,2, \ldots$ :

$$
\begin{aligned}
& \sum_{u=0}^{\infty} \frac{x^{u}}{u !}{ }^{p, q} \Phi_{h: k ; l ; m, n: r ; v ; w}^{\alpha: \beta ; \gamma ; \lambda, \mu: v ; \rho ; \sigma}(z, t, s-u, a) \\
= & { }^{p, q} \Phi_{h: k ; l ; m, n: r ; v ; w}^{\alpha: \beta ; \gamma, \mu: \gamma ; \rho}\left(z e^{p x}, t e^{q x}, s, a\right) e^{a x} .
\end{aligned}
$$


Proof. Replacing $s$ by $s-u$ in (1.7), multiply during by $x^{u} / u$ ! and then sum up, we obtain (4.4).

Theorem 4.5. The following result holds true:

$$
\begin{aligned}
& \sum_{i, j=0}^{\infty} \frac{\left[\left(\alpha_{h}\right)\right]_{2 i+j}\left[\left(\beta_{k}\right)\right]_{i+j}\left[\left(\gamma_{l}\right)\right]_{i}\left[\left(\lambda_{m}\right)\right]_{j}}{\left[\left(\mu_{n}\right)\right]_{2 i+j}\left[\left(v_{r}\right)\right]_{i+j}\left[\left(\rho_{v}\right)\right]_{i}\left[\left(\sigma_{w}\right)\right]_{j}} \frac{(p i+q j) z^{i} t^{j}}{i ! j !(a+p i+q j)^{s}} \\
& ={ }^{p, q} \Phi_{h: k ; \gamma ; m, n: r ; v ; w}^{\alpha: \beta ; \gamma, \mu: v ; \sigma}(z, t, s-1, a)-a^{p, q} \Phi_{h: k ; l ; m, n: r ; v ; w}^{\alpha: \beta ; \gamma ; \lambda, \mu: v ; \rho ; \sigma}(z, t, s, a) .
\end{aligned}
$$

Proof. Denote left hand side by $\Lambda$, so that

$$
\begin{aligned}
& a \Lambda=\sum_{i, j=0}^{\infty} \frac{\left[\left(\alpha_{h}\right)\right]_{2 i+j}\left[\left(\beta_{k}\right)\right]_{i+j}\left[\left(\gamma_{l}\right)\right]_{i}\left[\left(\lambda_{m}\right)\right]_{j}}{\left[\left(\mu_{n}\right)\right]_{2 i+j}\left[\left(v_{r}\right)\right]_{i+j}\left[\left(\rho_{v}\right)\right]_{i}\left[\left(\sigma_{w}\right)\right]_{j}} \frac{[(a+p i+q j)-(p i+q j)] z^{i} t^{j}}{i ! j !(a+p i+q j)^{s}} \\
& ={ }^{p, q} \Phi_{h: k ; \gamma ; m, n: r ; v ; w}^{\alpha: \beta ; \lambda, \mu: v ; \rho ; \sigma}(z, t, s-1, a)-\Lambda .
\end{aligned}
$$

yields the theorem.

\section{References}

[1] L. C. Andrews, Special Functions for Engineers and Applied Mathematician, Macmillan, New York, 1985.

[2] Maged G. Bin-Saad, Hypergeometric series associated with the Hurwitz-Lerch zeta function, Acta Math. Univ. Comenian. 78 (2009), 269-286.

[3] Maged G. Bin-Saad and Amani M. Hanballa, On power series associated with general family of the Hurwitz-Lerch zeta functions, Global J. Math. 2 (2015), 180-195.

[4] Maged G. Bin-Saad and Amani M. Hanballa, On hypergeometric series associated with the generalized zeta functions, SCIREA Journal of Mathematics 1 (2016), 53-62.

[5] Maged G. Bin-Saad, M. A. Pathan and Ali Z. Bin-Alhag, On multiple zeta function and associated properties, Turkish Journal of Analysis and Number Theory 6 (2018), 84-89. https://doi.org/10.12691/tjant-6-3-3

[6] J. Choi and R. K. Parmar, An extension of the generalized Hurwitz-Lerch zeta function of two variables, Filomat 31(1) (2017), 91-96. https://doi.org/10.2298/FIL1701091C 
[7] O. A. Daman and M. A. Pathan, A further generalization of the Hurwitz zeta function, Math. Sci. Res. J. 16(10) (2012), 251-259.

[8] A. Erdélyi, W. Magnus, F. Oberhettinger and F. G. Tricomi, Higher Transcendental Functions, Vol. I, McGraw-Hill Book Inc., New York, Toronto and London, 1953.

[9] H. Exton, A note on a hypergeometric transformation, Bull. Calcutta Math. Soc. 71 (1979), 337-340.

[10] H. Exton, Reducible double hypergeometric functions and associated integrals, An. Fac. Ci. Univ. Porto 63(1-4) (1982), 137-143.

[11] S. P. Goyal and R. K. Laddha, On the generalized Riemann zeta functions and the generalized Lambert function, Ganita Sandesh 11 (1997), 99-108.

[12] M. Katsurada, An application of the Mellin-Barnes type of integral to the mean square of l-function, Lith. Math. J. 38 (1998), 77-88. https://doi.org/10.1007/BF02465546

[13] G. Lauricella, Sulle funzioni ipergeometriche a piu variabili, Rend. Circ. Matem. 7 (1893), 111-158. https://doi.org/10.1007/BF03012437

[14] K. S. Nisar, Further extension of the generalized Hurwitz-Lerch Zeta function of two variables, 2017. arXiv:1706.03516v1[math.CA]

[15] K. S. Miller and B. Rose, An Introduction to The Fractional Calculus and Fractional Differential Equations, New York, 1993.

[16] M. A. Pathan and O. Daman, On Generalization of Hurwitz zeta function, Non-Linear World Journal, (2018), to appear.

[17] L. J. Slater, Confluent Hypergemetric Functions, Cambridge University Press, Cambridge, London and New York, 1960.

[18] H. M. Srivastava, A new family of the $\lambda$-generalized Hurwitz-Lerch Zeta functions with applications, Appl. Math. Inf. Sci. 8(4) (2014), 1485-1500.

https://doi.org/10.12785/amis/080402

[19] H. M. Srivastava and J. Choi, Zeta and q-Zeta Functions and Associate Series and Integrals, Elsevier Science, Publishers, Amsterdam, London and New York, 2012. https://doi.org/10.1016/B978-0-12-385218-2.00002-5

[20] H. M. Srivastava and P. W. Karlsson, Multiple Gaussian Hypergeometric Series, Halsted Press (Ellis Horwood Limited, Chichester), John Wiley and Sons, New York, Chichester, Brisbane, and Toronto, 1985. 
[21] H. M. Srivastava and H. L. Manocha, A Treatise on Generating Functions, Halsted Press (Ellis Horwood Limited, Chichester), John Wiley and Sons, New York, 1984.

[22] H. M. Srivastava and R. Panda, An integral representation for the product of two Jacobi polynomials, J. London Math. Soc. 12 (1976), 419-425.

https://doi.org/10.1112/j1ms/s2-12.4.419

[23] H. M. Srivastava and M. A. Pathan, Some bilateral generating functions for the extended Jacobi polynomials, Comment. Math. Uni. St. Paul 28(1) (1979), 23-30.

[24] H. M. Srivastava, M. J. Luo and R. K. Raina, New results involving a class of generalized Hurwitz-Lerch Zeta functions and their applications, Turkish Journal of Analysis and Number Theory 1(1) (2013), 26-35. https://doi.org/10.12691/tjant-1-1-7

[25] H. M. Srivastava, R. K. Saxena, T. K. Pogány and R. Saxena, Integral and computational representations of the extended Hurwitz-Lerch zeta function, Integral Transforms Spec. Funct. 22 (2011), 487-506. https://doi.org/10.1080/10652469.2010.530128 